\title{
Die EU und ihre Nachbarschaftsbeziehungen: etablierte Assoziierungsmodelle und neue Grundformen
}

\author{
Barbara Lippert
}

In this article, the author presents established models of association of the European Union (EU) with European third countries. She shows their different strategic perspectives, outlines benefits and problems, and examines the potential for developing these relations. Basically, these can go in the direction of expanding or dismantling partial sectoral integration. In addition, new basic forms of EU neighbourhood relations are discussed: the introduction of a new status of partial membership in the EU and inspired by the European Economic Area - the creation of a European political and economic area.

Zwischen 1989 und 2019 hat sich die Europäische Union (EU) durch die Aufnahme von 16 neuen Mitgliedstaaten tiefgreifend verändert. Parallel $\mathrm{zu}$ den Erweiterungsrunden von 1995, 2004 bzw. 2007 und 2013 entwickelte sie ihre Assoziierungs- und Kooperationsbeziehungen im Rahmen des Europäischen Wirtschaftsraums (EWR) zu Staaten der Europäischen Freihandelsassoziation (EFTA), im Rahmen des Stabilisierungs- und Assoziierungsprozesses (SAP) zu Westbalkanländern und im Rahmen der Europäischen Nachbarschaftspolitik (ENP) zu 16 Ländern in Osteuropa und der Mittelmeerregion. Mithilfe dieser unterschiedlichen Beziehungsmodelle strukturierte die EU ihre Nachbarschaftsräume mit mehr oder weniger großem Erfolg. Diese Modelle und ihre praktischen Ausführungen durch bilaterale Beziehungen sind gegenwärtig in unterschiedlichem Maß und von verschiedenen Seiten einem erheblichen Leistungs- und Legitimitätsdruck ausgesetzt: Die ohnehin ruhenden Beitrittsverhandlungen mit der Türkei stehen vor einem offiziellen Aus und für die Länder des Westbalkans sieht die EU eher auf lange denn auf mittlere Sicht eine Aufnahme vor. 15 Jahre nach ihrer Einführung hat die EU die ENP mehr und mehr von der ursprünglich der Erweiterungspolitik nachgebildeten Strategie gelöst und sie den traditionellen Außenbeziehungen angenähert. Als vergleichsweise funktionsfähig hat sich der aus einer EU- und einer EFTA-Säule bestehende EWR erwiesen. Dabei ist jedoch die EFTA-Säule ein exklusiver Club aus Norwegen, Liechtenstein und Island geblieben, in den z.B. ehemalige EU-Mitglieder nicht einfach hinüberwechseln können. Der Austritt des Vereinigten Königreichs aus der EU beendet voraussichtlich 2019 die lineare Erweiterungsdynamik (immer mehr Mitglieder) und hat damit Schwung in die Debatte über alte und neue Beziehungsmodelle der EU gebracht. Im Zusammenhang mit den Brexit-Verhandlungen wurde auch auf dem Kontinent diskutiert, wie flexibel, kreativ und mit welcher politischen Vision die EU mit ihrem künftigen Nachbarn verhandeln sollte. Es besteht also Anlass, Vertragsgrundlagen, strategische Perspektiven sowie Kosten und Nutzen der jeweiligen Modelle kritisch zu bewerten und deren Entwicklungsmöglichkeiten auszuloten bzw. neue Modelle zu entwerfen.

* Dr. Barbara Lippert, Forschungsdirektorin der Stiftung Wissenschaft und Politik, Berlin. 


\section{Architekturen der Nachbarschaft: Grundformen und Bausteine}

Die EU gründet ihre Beziehungen zu Drittstaaten vorzugsweise auf Abkommen, die zwischen beiden Parteien ein langfristiges Kooperations- oder Assoziierungsverhältnis schaffen. ${ }^{1}$ Sie stützt diese überwiegend auf Art. 217 (Assoziierung) des Vertrags über die Arbeitsweise der Europäischen Union (AEUV), außerdem dort auf Art. 207 (gemeinsame Handelspolitik), Art. 212 (Zusammenarbeit mit Drittstaaten) und Art. 218 (Übereinkünfte mit Drittstaaten). Mit diesen Grundformen und den dazugehörigen Bausteinen hat die EU eine Fülle von Ausführungsvarianten geschaffen. Zum klassischen Programm der Assoziierung zählen folgende Bausteine: die Herstellung von freiem Verkehr v.a. von Gütern (teils auch Dienstleistungen), die Übernahme oder gegenseitige Anerkennung von Standards, die Einbindung von Assoziierten in Programme und Agenturen der EU sowie in einzelne EU-Politiken und andere Formen des Zusammenwirkens wie der politische Dialog im Bereich der Außenpolitik oder finanzielle und technische Unterstützung. ${ }^{2}$ Charakteristika der Assoziierung sind die politische Substanz im Sinne gegenseitiger Rücksichtnahme, die meist schon in der Präambel der Abkommen in Motiv- und Zielsetzungen angesprochen wird, die Dauerhaftigkeit der Beziehungen im Sinne institutioneller Verfestigung, die Bilateralität und die formale Parität bei gegenseitigen Rechten und Pflichten. ${ }^{3}$ Die Abkommen sind dynamisch angelegt, da die Assoziierungsinstitutionen laut dem Assoziierungsartikel das "gemeinsame Vorgehen“ und die „besonderen Verfahren“ für die Weiterentwicklung der Beziehungen beschließen können. Alles in allem haben Assoziierte eine privilegierte Beziehung zur EU und nehmen partiell am Gemeinschaftssystem teil. Das führt zu Teilintegration ohne Mitgliedschaft, die ohnehin nur für europäische Länder infrage kommt (Art. 49 des Vertrags über die Europäische Union, EUV). Charakteristisch ist jedoch, dass die EU die Architektin der Beziehungen ist, die die Baupläne bestimmt und die Normen setzen will. Letzteres zeigt sich besonders stark bei der Regelung des Zugangs zum EU-Binnenmarkt. Die EU beharrt aus integrationspolitischen Gründen darauf, dass die vier Freiheiten für Güter, Dienstleistungen, Kapital und ArbeitnehmerInnen unauflöslich zusammengehören und nur als Paket gewährt werden. So hielten die verbleibenden 27 Mitgliedstaaten in den Verhandlungen mit dem Vereinigten Königreich immer wieder fest, „dass die vier Freiheiten unteilbar sind und es kein ,Rosinenpicken' geben kann, d. h. eine Beteiligung am Binnenmarkt lediglich in einzelnen Sektoren, die die Integrität und das ordnungsgemäße Funktionieren des Binnenmarktes untergraben würde“. 4 Die Beziehung zwischen europäischen Vertragsparteien ist in der Regel asymmetrisch zum Vorteil der EU. ${ }^{5}$ Das ist selbst beim EWR der Fall und zeigte sich bereits im Vorlauf zu möglichen Verhand-

1 Siehe dazu auch Barbara Lippert: Beitritt, Austritt und europäische Assoziierung - Vertragsgrundlagen und politische Praxis der Europäischen Union, in: Peter Becker/Barbara Lippert (Hrsg.): Handbuch Europäische Union, Wiesbaden 2019, S. $1 \mathrm{ff}$.

2 So bei Rudolf Mögele: Art. 217 AEUV, in: Rudolf Streinz (Hrsg.): EUV/AEUV. Vertrag über die Europäische Union, Vertrag über die Arbeitsweise der Europäischen Union, Charta der Grundrechte der Europäischen Union, 3. Auflage, München 2018, hier S. 1999.

3 Vgl. Thomas Oppermann/Claus Dieter Classen/Martin Nettesheim: Europarecht. Ein Studienbuch, 7. Auflage, München 2016, S. 666f.; Mögele: Art. 217 AEUV, 2018, S. $2000 f f$.

4 Europäischer Rat: Europäischer Rat (Artikel 50) (Tagung am 23. März 2018) - Leitlinien, EUCO XT 20001/18.

5 Wirtschaftlich schwächeren Staaten bietet die EU Entwicklungsanreize, bspw. durch einseitige Marktöffnung. Abkommen mit ökonomisch ebenbürtigen Drittstaaten wie Kanada oder Japan haben einen engeren Zuschnitt oder kommen erst gar nicht zustande wie im Fall Russlands und der USA. 
lungen mit dem Vereinigten Königreich über eine „ambitionierte, breite, vertiefte und flexible Partnerschaft". 6

Im Umfeld der großen Osterweiterung 2004 und der Arbeiten des Verfassungskonvents ist erstmals im EU-Primärrecht ein Referenzpunkt speziell für die Beziehungen zu Nachbarn geschaffen worden: der Nachbarschaftsartikel 8 EUV. Mit diesem Artikel (siehe auch unten) ist jedoch keine neue Grundform geschaffen worden, die z.B. zwischen Assoziierung und Mitgliedschaft angesiedelt wäre. Die EU hat ihr Angebot an Drittstaaten nicht substanziell erweitert. Vielmehr ist, nicht ganz ungewollt, eine gewisse Unschärfe in die bestehenden Angebote gekommen. Vage ist von „speziellen Übereinkünften“ mit „Ländern in ihrer Nachbarschaft“ die Rede. Die geografische Reichweite der Nachbarschaft oder gar einzelne Länder bleiben unbestimmt. Sinn und Zweck des Artikels erschließen sich am ehesten, wenn dessen Subtext - die bei der Verabschiedung des Vertrags von Lissabon bereits lancierte ENP - mitgelesen wird. Jedoch hat die EU diesen Artikel bisher weder rechtlich noch politisch für die Gestaltung der Beziehungen zu den Nachbarn im Osten und Süden genutzt. Art. 8 EUV ist stark an Art. 217 AEUV angelehnt und von diesem und anderen materiellen Bestimmungen im AEUV abhängig. Im Mittelpunkt steht die Kooperation - der Begriff „Assoziierung“ taucht nicht auf. Ebenso wenig werden „besondere Verfahren“ (Art. 217 AEUV) erwähnt. Ähnlich wie im Falle der Assoziierung ist jedoch von gegenseitigen Rechten und Pflichten sowie der Möglichkeit eines gemeinsamen Vorgehens die Rede. Außerdem sind nicht näher bestimmte, regelmäßige Konsultationen vorgesehen, worunter etwa der politische Dialog im Rahmen der Gemeinsamen Außen- und Sicherheitspolitik (GASP) oder aber andere Dialogformate rangieren können. Art. 8 EUV hat weder für die EU noch für Drittstaaten neue politische und rechtliche Optionen zur Gestaltung der bi- und multilateralen Beziehungen eröffnet. Die EU hat nicht einmal das politische Kapital des Art. 8 EUV genutzt, um nach dem Vorbild der Europaabkommen mit den ostmitteleuropäischen Ländern der 1990er Jahre einen besonderen Typus von Assoziierungsabkommen symbolisch aufzuwerten, also bspw. die Assoziationsabkommen (AA) mit der Ukraine oder Georgien als „Nachbarschaftsabkommen“ zu qualifizieren.

\section{Strategische Perspektiven und Zwischenbilanzen}

Die Assoziierungsabkommen, die die EU mit Nachbarn in Europa (siehe Tabelle 1) und dem Mittelmeerraum geschlossen hat, unterscheiden sich zunächst in ihrer strategischen Perspektive, also ob sie als Vorstufe oder Alternative zur Mitgliedschaft angelegt sind. ${ }^{7}$ Darüber hinaus divergieren sie hinsichtlich beabsichtigtem Umfang und Grad der Konvergenz mit dem EU-Acquis.

6 Rat der Europäischen Union: Politische Erklärung zur Festlegung des Rahmens für die künftigen Beziehungen zwischen der Europäischen Union und dem Vereinigten Königreich. Brüssel, 22. November 2018, XT 21095/18, S. 2.

7 Hier werden die Abkommen mit Mittelmeerländern nicht behandelt. Vgl. die Beiträge zur ENP und zu Maghreb- bzw. Maschrek-Ländern in: Tobias Schumacher/Andreas Marchetti/Thomas Demmelhuber (Hrsg.): The Routledge Handbook on the European Neighbourhood Policy, London/New York 2018, S. 324ff. 


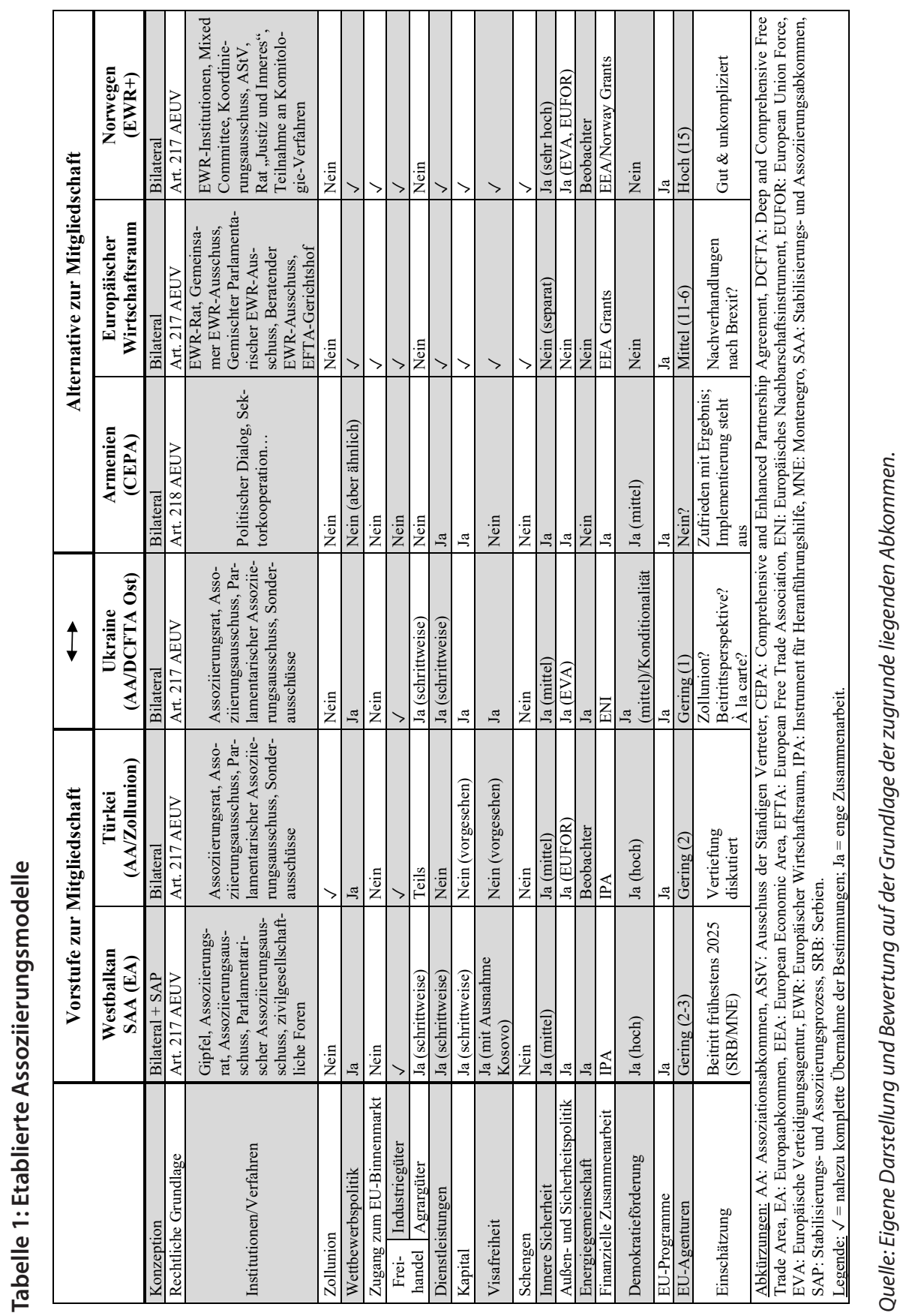


Diese partielle, sektorale Integration geschieht auf dem Wege der wechselseitigen Anerkennung oder Harmonisierung durch Übernahme des EU-Rechts, des Sich-Anschließens an EU-Positionen und der Nachbildung von EU-Regeln. Entgegen der Vorstellung aus der Hallstein-Zeit, Assoziierung könnte „Mitgliedschaft minus ein Prozent“ bedeuten, ${ }^{8}$ hat die EU in allen Beziehungen zu Drittstaaten eine scharfe Integrationsgrenze aufrechterhalten. Danach werden Drittstaaten nicht an EU-Entscheidungen beteiligt und sind nicht in EUOrganen vertreten. Insofern gibt es keinen Status der Teilmitgliedschaft. Die politische Schmerzgrenze zieht die EU bei der Unauflöslichkeit der vier Freiheiten, bei der Mitgliedschaft in der Währungsunion und z.B. bei der Einbeziehung in Verteilungsmechanismen (Gemeinsame Agrarpolitik, Strukturpolitik). Bislang deutet sich bei der EU noch kein Politikwechsel an. So sind Vorschläge wie die auf die Balkanländer gemünzte Junior-Mitgliedschaft oder eine kontinentale Partnerschaft zwischen der EU und Großbritannien ins Leere gelaufen und zu keiner Zeit Verhandlungsgegenstand geworden (siehe Tabelle 2). Mit ihrer Ablehnung der sogenannten Rosinenpickerei durch London liefert die EU eine integrationspolitische Begründung und keine rein funktional-wirtschaftliche. Sie zieht demnach ebenso rote Linien wie die britische Regierung. ${ }^{9}$

Die Beziehungen zu europäischen Nachbarn stellen die EU immer vor besondere Fragen, da diese gemäß Art. 49 EUV einen Antrag auf Mitgliedschaft stellen können. Diesem muss nicht zwingend irgendeine Vertragsbeziehung vorausgehen. Dennoch ist relevant, ob die angestrebten Vertragsbeziehungen als Vorstufe bzw. Durchgangsstadium oder aber als Alternative zur Mitgliedschaft konzipiert sind. Im ersten Fall stehen der Heranführungsprozess und seine Gestaltung im Mittelpunkt. Daran sind oft auch Fragen des Status gebunden, mit dem die Anwartschaft auf Mitgliedschaft zum Ausdruck kommen soll. Hier hat die EU viel Spielraum, denn gängige Etikettierungen als „privilegiert“, „umfassend“, „tief und umfassend“ etc. liegen im Feld der Diplomatie und politischen Kommunikation, aber kaum des Rechts. ${ }^{10}$ Kompliziert wird das bilaterale Vertragsverhältnis, wenn EU und Drittstaat unterschiedliche strategische Ziele verfolgen oder sich (z.B. wegen interner Spaltungen) ambivalent verhalten - wie es die EU über Jahrzehnte gegenüber der Türkei tat.

Betrachtet man die unterschiedlichen Assoziierungen im Hinblick auf die Fähigkeit zur Übernahme des EU-Acquis, so lassen sich die assoziierten Länder in leistungsstarke und leistungsschwache gruppieren. Leistungsstarke Länder finden sich überwiegend unter denen, für die die Assoziierung eine Alternative zur Mitgliedschaft ist; leistungsschwache Länder sehen die Assoziierung als Vorbereitung auf den Beitritt und die EU sieht darin ein Heranführungsinstrument an die spätere (jedoch nicht garantierte) Mitgliedschaft.

8 David Phinnemore: Association: Stepping-stone or Alternative to EU Membership?, Sheffield 1999, S. 23.

9 Europäischer Rat: Leitlinien, März 2018; Rede der britischen Premierministerin Theresa May zu Großbritanniens Austritt aus der Europäischen Union, 17.1.2017 im Lancester House, abrufbar unter: https://www.gov.uk/g overnment/speeches/the-governments-negotiating-objectives-for-exiting-the-eu-pm-speech.de (letzter $\mathrm{Zu}$ griff: 3.5.2019).

10 „Privilegiert“: EU-Tunesien-Beziehungen; „umfassend“: EU-Armenien-Beziehungen; „tief und umfassend“: DCFTA mit der Ukraine, Moldau und Georgien. 
Tabelle 2: Weitere Modelle in der Diskussion

\begin{tabular}{|c|c|c|c|c|c|}
\hline & \multicolumn{2}{|c|}{ Teilintegration } & \multicolumn{2}{|c|}{ Teilmitgliedschaft } \\
\hline & & \begin{tabular}{|c|} 
Vereinigtes \\
Königreich \\
„ambitionierte, \\
breite, vertiefte und \\
flexible \\
Partnerschaft"“11
\end{tabular} & $\begin{array}{c}\text { Europäischer } \\
\text { Politik- und } \\
\text { Wirtschaftsraum }^{12}\end{array}$ & $\begin{array}{l}\text { Kontinentale } \\
\text { Partnerschaft }\end{array}$ & $\begin{array}{c}\text { Junior- } \\
\text { Mitgliedschaft }^{14}\end{array}$ \\
\hline \multicolumn{2}{|c|}{ Konzeption } & Bilateral & $\begin{array}{l}\text { Multilateral/ } \\
\text { zwei Pfeiler }\end{array}$ & Bilateral & Bilateral \\
\hline \multicolumn{2}{|c|}{ Rechtliche Grundlage } & $\begin{array}{l}\text { Art. } 50 \text { EUV, } \\
\text { Art. } 218 \text { AEUV }\end{array}$ & $\begin{array}{l}\text { Art. } 217 \text { AEUV für } \\
\text { Länder mit bilatera- } \\
\text { ler EU-Anbindung } \\
\text { (AA/DCFTA) }\end{array}$ & Art. 217 AEUV & Art. 49 EUV plus \\
\hline \multicolumn{2}{|c|}{ Institutionen/Verfahren } & $\begin{array}{l}\text { Assoziationsabkom- } \\
\text { men, regelmäßige } \\
\text { Dialoge, Konsulta- } \\
\text { tionen, Parlamenta- } \\
\text { rischer Ausschuss, } \\
\text { Sonderausschüsse, } \\
\text { Joint Committee }\end{array}$ & $\begin{array}{l}\text { EPWR-Rat, Gemein- } \\
\text { samer EPWR-Aus- } \\
\text { schuss, Gemischter } \\
\text { Parlamentarischer } \\
\text { EPWR-Ausschuss, } \\
\text { Beratender EPWR- } \\
\text { Ausschuss }\end{array}$ & $\begin{array}{l}\text { Intergouvernementale } \\
\text { Struktur, Partner- } \\
\text { schaftsrat, Mitent- } \\
\text { scheidungsrecht Dritt- } \\
\text { staat, Konsultation bei } \\
\text { EU-Gesetzgebung, in- } \\
\text { tensiver als EWR }\end{array}$ & $\begin{array}{l}\text { EU-Mitgliedschaft } \\
\text { „2. Klasse“ (Annä- } \\
\text { herung), Beteili- } \\
\text { gung und Mitent- } \\
\text { scheidung an EU- } \\
\text { Institutionen, kon- } \\
\text { zentrische Struktur }\end{array}$ \\
\hline \multicolumn{2}{|c|}{ Zollunion } & $\begin{array}{l}\text { Nein } \\
\text { (aber Backstop) }\end{array}$ & Option & Nein & Ja \\
\hline \multicolumn{2}{|c|}{ Wettbewerbspolitik } & $\mathrm{Ja}$ & $\checkmark$ & $\mathrm{Ja}$ & Schrittweise \\
\hline \multicolumn{2}{|c|}{$\begin{array}{l}\text { Zugang zum EU-Bin- } \\
\text { nenmarkt }\end{array}$} & Nein & $\checkmark$ & $\begin{array}{l}\checkmark \text { (ohne Arbeitneh- } \\
\text { merfreizügigkeit) }\end{array}$ & Langfristig \\
\hline \multirow{2}{*}{$\begin{array}{l}\text { Frei- } \\
\text { handel }\end{array}$} & Industriegüter & Ja & $\checkmark$ & Ja & Ja \\
\hline & Agrargüter & Nein & Nein/Option & $\mathrm{Ja}$ & Schrittweise \\
\hline \multicolumn{2}{|c|}{ Dienstleistungen } & $\begin{array}{l}\text { „building on recent } \\
\text { FTAs“ }\end{array}$ & $\checkmark$ & Ja & Schrittweise \\
\hline \multicolumn{2}{|l|}{ Kapital } & $\begin{array}{l}\text { Nein, enge Koopera- } \\
\text { tion }\end{array}$ & $\checkmark$ & Ja & Schrittweise \\
\hline \multicolumn{2}{|c|}{ Visafreiheit } & $\mathrm{Ja}$ & $\checkmark$ & Ja & Ja \\
\hline \multicolumn{2}{|c|}{ Schengen } & Nein & $\checkmark$ & Nein & Perspektivisch \\
\hline \multicolumn{2}{|c|}{ Innere Sicherheit } & Ja (hoch) & Nein & $\mathrm{Ja}$ & Schrittweise \\
\hline \multicolumn{2}{|c|}{$\begin{array}{l}\text { Außen- und Sicher- } \\
\text { heitspolitik }\end{array}$} & Ja (hoch) & Ja & $\mathrm{Ja}$ & $\mathrm{Ja}$ \\
\hline \multicolumn{2}{|c|}{ Energiegemeinschaft } & Zusammenarbeit & $\mathrm{Ja}$ & $\mathrm{Ja}$ & Schrittweise \\
\hline
\end{tabular}

11 Rat der Europäischen Union: Politische Erklärung zur Festlegung des Rahmens für die künftigen Beziehungen zwischen der Europäischen Union und dem Vereinigten Königreich, 2018.

12 Barbara Lippert: Assoziierung plus gesamteuropäische Aufgabenkonföderation: Plädoyer für eine selbstbewusste Nachbarschaftspolitik der EU, in: integration 2/2006, S. 149ff.; Barbara Lippert: Teilhabe statt Mitgliedschaft? Die EU und ihre Nachbarn im Osten, in: osteuropa 2-3/2007, S. 69ff.

13 Jean Pisani-Ferry et al.: Europe after Brexit: A proposal for a continental partnership, Bruegel: External Publication, 29. August 2016.

14 Franz-Lothar Altmann: EU und Westlicher Balkan. Von Dayton nach Brüssel: ein allzu langer Weg?, Stiftung Wissenschaft und Politik: SWP-Studie S 1/2005; Andrew Duff: On Governing Europe, London 2012, S. 68ff., abrufbar unter: https://www.federalists.eu/fileadmin/files_uef/Federalists_Publications/On_Governing_Europ e_Andrew_Duff_2012.pdf (letzter Zugriff: 7.2.2019). 


\begin{tabular}{|c|c|c|c|c|}
\hline & \multicolumn{2}{|c|}{ Teilintegration } & \multicolumn{2}{|c|}{ Teilmitgliedschaft } \\
\hline & $\begin{array}{c}\text { Vereinigtes } \\
\text { Königreich } \\
\text { "ambitionierte, } \\
\text { breite, vertiefte und } \\
\text { flexible } \\
\text { Partnerschaft“ }\end{array}$ & $\begin{array}{l}\text { Europäischer } \\
\text { Politik- und } \\
\text { Wirtschaftsraum }\end{array}$ & $\begin{array}{l}\text { Kontinentale } \\
\text { Partnerschaft }\end{array}$ & $\begin{array}{c}\text { Junior- } \\
\text { Mitgliedschaft }\end{array}$ \\
\hline $\begin{array}{l}\text { Finanzielle Zusammen- } \\
\text { arbeit }\end{array}$ & Geber & Ja & Geber & EU-Fonds \\
\hline Demokratieförderung & Nein & $\mathrm{Ja}$ & Ja & Bei Bedarf \\
\hline EU-Programme & Ja & $\mathrm{Ja} /$ Konditionalität & Ja & Ja \\
\hline EU-Agenturen & $\mathrm{Ja}$ & $\mathrm{Ja}$ & $\mathrm{Ja}$ & Ja \\
\hline Einschätzung & $\begin{array}{l}\text { Neuer Typ von As- } \\
\text { soziierung und Part- } \\
\text { nerschaft }\end{array}$ & $\begin{array}{l}\text { Neue Grundform, } \\
\text { die auf die ÖP auf- } \\
\text { bauen könnte }\end{array}$ & $\begin{array}{l}\text { Neue Grundform, } \\
\text { Politikwechsel und } \\
\text { Vertragsänderung }\end{array}$ & $\begin{array}{l}\text { Neue Grundform, } \\
\text { erfordert Vertrags- } \\
\text { änderung }\end{array}$ \\
\hline \multicolumn{5}{|c|}{$\begin{array}{l}\text { Abkürzungen: AA: Assoziationsabkommen, DCFTA: Deep and Comprehensive Free Trade Area, EPWR: Euro- } \\
\text { päischer Politik- und Wirtschaftsraum, EWR: Europäischer Wirtschaftsraum, FTA: Free Trade Agreement, ÖP: } \\
\text { Östliche Partnerschaft. } \\
\text { Legende: } \checkmark=\text { nahezu komplette Übernahme der Bestimmungen; Ja = enge Zusammenarbeit. }\end{array}$} \\
\hline
\end{tabular}

Quelle: Eigene Darstellung und Bewertung auf Basis der angegebenen Quellen.

Länder, die prinzipiell beitrittsfähig sind, aber ausdrücklich eine Alternative zur Mitgliedschaft suchen, kommen derzeit vor allem aus dem Kreis der EFTA und gehören teils auch dem EWR an (siehe Tabelle 1). Sie sind an der wirtschaftlichen Integration interessiert, aber höchstens partiell an der politischen. Die zugehörigen bilateralen Vertragswerke sind nicht statisch, die Parteien entwickeln sie durch Ergänzungen und Revision fort. Sie bleiben aber bewusst unterhalb der Mitgliedschaft. Die Vertragswerke funktionieren überwiegend gut und werden beiderseits als gewinnbringend eingeschätzt. Nur den leistungsstarken assoziierten Ländern gewährt die EU die volle Teilhabe an den vier Freiheiten des Binnenmarkts, ergänzt z.B. um die Integration in den Schengen-Raum wie im Falle Norwegens. Dieses Modell gilt als das „High End“ der Assoziierung. Die Probleme liegen für die Drittstaaten in Fragen der Input-Legitimität, denn sie haben keine Entscheidungsrechte und ihr politischer Einfluss auf die Politikformulierung wird durch Konsultation und Vertretung in Vorbereitungs- und Durchführungsgremien beschränkt. Sie sind auch in höherem Maße als Mitglieder der Unberechenbarkeit der allgemeinen Integrationsentwicklung ausgesetzt, ohne darauf Einfluss nehmen zu können. ${ }^{15}$ So sind die EFTA-Mitglieder im EWR direkt vom Austritt des Vereinigten Königreichs aus der EU betroffen. Man denke an die Regelungen zur Freizügigkeit. ${ }^{16}$ In der Brexit-Debatte wurde für Großbritannien ein Modell „Norwegen plus“ ins Spiel gebracht, das u.a. verlangen würde, dass Großbritannien

15 Vgl. Norwegisches Außenministerium: Official Norwegian Reports NOU 2012:2 Chapter 1. Outside and Inside. Norway's agreements with the European Union.

16 So haben die EFTA-Staaten mit dem Vereinigten Königreich ein eigenes Abkommen geschlossen, um mit dem Austritt zusammenhängende Fragen, insbesondere Residenzrechte, zu klären. Siehe Regierung des Vereinigten Königreichs (Hrsg.): Agreement on arrangements between Iceland, the Principality of Liechtenstein, the Kingdom of Norway and the United Kingdom of Great Britain and Northern Ireland following the withdrawal of the United Kingdom from the European Union, the AA Agreement and other agreements applicable between the United Kingdom and the EEA EFTA States by virtue of the United Kingdom's membership of the European Union, 20. Dezember 2018. 
der EFTA und dann dem EFTA-Pfeiler im EWR beitritt. Prinzipiell könnte man sich auch vorstellen, dass Großbritannien einen eigenen dritten Pfeiler des EWR bildet. Großbritannien würde seinen Sonderstatus im EWR ähnlich wie als EU-Mitglied mit Opt-in- und Opt-out-Rechten etablieren wollen. Erhielte dann Großbritannien bessere Konditionen als die EFTA-Länder, wäre die Kompatibilität zwischen den verschiedenen Assoziierungsverhältnissen, die die EU mit Nachbarn eingegangen ist, gefährdet. Einzelne Elemente aus dem EWR, wie der EFTA-Gerichtshof zur Klärung von Streitigkeiten zwischen den Parteien, könnten jedoch auch für das künftige Verhältnis zu Großbritannien interessant sein. ${ }^{17}$

Eine größere Anzahl von assoziierten Ländern in der europäischen Nachbarschaft ist in absehbarer Zeit nicht beitrittsfähig, strebt aber oftmals die Mitgliedschaft an und benötigt dafür die Unterstützung der EU. Die EU hält im Rahmen des erneuerten Konsenses über die Erweiterungspolitik ${ }^{18}$ am Konzept der Vollmitgliedschaft fest. Sie will aus Kandidaten perfekte Mitglieder im Sinne der Kopenhagener Beitrittskriterien machen. In der Realität bedeutet das unabsehbar lange Heranführungsperioden, die dann wie 2007 im Fall von Rumänien und Bulgarien durch vorzeitige Aufnahme beendet werden und beträchtliche Integrationskosten in der EU verursachen können. Dafür stehen die Stichworte Divergenz, Heterogenität, Differenzierung und Überdehnung. Der Umgang mit diesen Ländern ist für die EU schwierig. Denn diese sind - im Lichte der Normhürden der Art. 8 und 49 EUV durch teils gravierende politische und wirtschaftliche Rückstände gekennzeichnet. Zudem ist ihre wirtschaftliche Bedeutung (mit Ausnahme der Türkei) für die EU insgesamt gering, aber ihre sicherheitspolitische und geostrategische erheblich, wie z.B. im Fall der Ukraine oder der Westbalkanländer.

Die EU konzipiert für die osteuropäischen Nachbarn unter dem Dach der Assoziierung Transformations- und Heranführungsprogramme umfassender Art und mit der Perspektive der immer engeren wirtschaftlichen Integration und politischen Assoziierung bis an die o.g. Integrations- und Schmerzgrenzen. Aus Gründen politischer Symbolik und bürokratischer Logik wurde dafür ein „Erweiterung light“-Ansatz gewählt, der eine Politik, Wirtschaft und Gesellschaft umfassende Transformation und Europäisierung verfolgt und entsprechend tiefe und umfassende Abkommen (DCFTA) gebiert. ${ }^{19}$ Praktisch wird dieser „alles umfassende Ansatz“ jedoch ausgehöhlt: durch dosierte oder arbiträre Konditionalität (orientiert an Demokratie - Good Governance - Resilienz) seitens der EU bzw. durch selektive Implementierung und zu schwache Ownership auf Seiten der Partner. ${ }^{20}$ Das Abkommen mit Armenien fällt notgedrungen weniger tief und umfangreich aus. ${ }^{21}$ Allerdings lassen sich auch Gründe für weniger umfassende Abmachungen finden, die sich stärker auf

17 Carl Baudenbacher: Totgesagte leben länger. 25 Jahre Europäischer Wirtschaftsraum: Was Großbritannien von der Efta lernen kann, in: Frankfurter Allgemeine Zeitung, 27. April 2017.

18 Rat der Europäischen Union: Europäischer Rat (Brüssel). 14./15. Dezember 2006. Schlussfolgerungen des Vorsitzes, Dok. 16879/1/06 REV1.

19 Kai-Olaf Lang/Barbara Lippert: Zur Neuausrichtung der ENP: Ein Liga-Modell nachbarschaftlicher Kooperation, in: Annegret Bendiek/Barbara Lippert/Daniela Schwarzer (Hrsg.): Entwicklungsperspektiven der EU. Herausforderungen für die deutsche Europapolitik, Stiftung Wissenschaft und Politik: SWP-Studie S 18/2011, S. $102 \mathrm{ff}$.

20 Susan Stewart: Die Stimmungslage in der Ukraine. Die politische und gesellschaftliche Instabilität wächst, Stiftung Wissenschaft und Politik: SWP-Aktuell 6/2018; Michael Emerson/Veronika Movchan (Hrsg.): Deepening EU-Ukrainian Relations. What, why and how?, 2. Auflage, London/Maryland 2018.

21 Franziska Smolnik: CEPA im „Neuen Armenien“. Armeniens Reformkurs unter der Regierung Paschinjan und das neue Partnerschaftsabkommen mit der EU, Stiftung Wissenschaft und Politik: SWP-Aktuell 42/2018. 
mittelfristig erreichbare Ziele konzentrieren. Andere Bereiche, wie etwa das Wettbewerbsrecht, können ausgeklammert oder zurückgestellt werden, weil sie weniger der Interessenlage und Leistungskraft von Ländern wie Georgien entsprechen. ${ }^{22}$

Da die Stabilisierungs- und Assoziierungsabkommen mit den Westbalkanländern und die Assoziierung/Zollunion mit der Türkei in Beitrittsverhandlungen und eine Heranführungsstrategie eingebettet sind, unterscheiden sie sich gegenwärtig nicht nur materiell von den DCFTA mit osteuropäischen Staaten, sondern v.a. strategisch. Die Hebel- und Transformationskraft einer Ausweitung der politischen Beitrittsperspektive ist jedoch nicht eindeutig und politisch umstritten. ${ }^{23}$

\section{Entwicklungsmöglichkeiten: Ausbau oder Rückbau}

Die heutige Vielfalt der Assoziierungs- und Partnerschaftsbeziehungen spricht dafür, dass die EU weiterhin länderspezifische Lösungen sucht, aber doch die regionalen Kontexte und geopolitischen Konstellationen berücksichtigen muss. Insofern kommen individuelle Optionen für die künftigen Beziehungen zu einzelnen Ländern und keine Einheitslösungen infrage. Ausgehend von den etablierten Assoziierungsmodellen können vier Entwicklungsmöglichkeiten ausgelotet werden.

\section{Erweiterung des EWR}

Beim bisher engsten Partnerschaftsverhältnis zwischen der EU und Drittstaaten, dem EWR, neigt die EFTA-Seite wohl nicht zur Erweiterung dieser Säule, wie die allseitige Reserviertheit im Fall des Vereinigten Königreichs verdeutlicht. ${ }^{24}$ Die Mitgliedschaft im EWR ist auch für osteuropäische Drittstaaten wenig interessant. Er bietet Ländern wie der Ukraine zu wenig politische Anbindung und Transformationsunterstützung und fordert keine geringere Binnenmarktreife als die EU-Mitgliedschaft. Attraktiv für Länder der Östlichen Partnerschaft (ÖP) - wie auch für die Türkei - wären am EWR wohl vor allem die Personenfreizügigkeit und wie im Fall Norwegens die Möglichkeit, in weitere Politikfelder oder Politikräume der EU eingebunden zu werden. Es wäre also eher an eine um Transformationshilfen ergänzte eigene Organisationsform, wie einen Europäischen Politik- und Wirtschaftsraum (siehe unten), zu denken.

\section{Optimierung der DCFTA/AA}

Die bestehenden DCFTA/AA könnten in zwei unterschiedliche Richtungen entwickelt werden: Man könnte die jeweiligen bilateralen Vertragsbeziehungen zu einer „ÖP plus“ anreichern (wie im Europäischen Parlament diskutiert) ${ }^{25}$ oder diese eher verschlanken und in Richtung „à la carte“ entwickeln. In beiden Fällen sind Maßnahmen und Formate zu über-

22 László Bruszt/Julia Langbein: Varieties of dis-embedded liberalism. EU integration strategies in the Eastern peripheries of Europe, in: Journal of European Public Policy 2/2017, S. $297 \mathrm{ff}$.

23 Barbara Lippert: Grenzwertig: die Fortsetzung der EU-Erweiterung als Stabilitätsexport, in: integration 2/2012, S. $123 \mathrm{ff}$.

24 Patrick Wintour: Norwegian politicians reject UK's Norway-plus Brexit plan, in: The Guardian, 7. Dezember 2018.

25 Europäisches Parlament: Östliche Partnerschaft: Gipfeltreffen im November 2017. Empfehlung des Europäischen Parlaments vom 15. November 2017 an den Rat, die Kommission und den EAD zur Östlichen Partnerschaft im Vorfeld des Gipfeltreffens im November 2017, P8_TA(2017)0440. 
legen, die die Implementierungsprozesse begleiten und unterstützen und so eine flexible Steuerung ermöglichen würden.

Im Fall der Türkei, die als einziger assoziierter Drittstaat auch eine Zollunion mit der EU bildet, ist eine „vertiefte Zollunion“26 in der Diskussion. So könnte der Agrar- und Dienstleistungssektor miteinbezogen werden. Für die Ukraine sind die Ergänzung des AA/ DCFTA um eine Zollunion oder gar der Beitritt zu Schengen (siehe Norwegen) diskutiert worden. ${ }^{27}$ Allerdings sind erhebliche Zweifel am wirtschaftlichen und gesellschaftlichen Nutzen solcher Ausbaupläne für die Drittstaaten anzumelden. Deren Motive liegen zumeist in der angestrebten politisch-symbolischen Aufwertung des Landes. Demgegenüber könnte ein Zurückstutzen des Umfangs der Assoziierung auf ausgewählte Bereiche oder Ziele, insbesondere ein Umgang „à la carte“ bei der Übernahme des Acquis, dem Ziel der wirtschaftlichen Entwicklung und des Wachstums dienlich sein. Damit könnten die Assoziierten diverse Beziehungen zu relevanten Partnern (Organisation für wirtschaftliche Zusammenarbeit und Entwicklung, Weltbank) entwickeln. Im Fall Armeniens entspricht dies auch dem Streben nach einer multivektoriellen Außenpolitik. Die Legitimität und Professionalität sowie das Interessenkalkül der Verhandlungspartner sind für solche Aushandlungsprozesse relevant, wie auch die Flexibilität der EU (etwa beim Protektionismus im Agrarhandel).

\section{Sockelvereinbarungen}

Gäbe die EU den Ansatz umfassender Rahmenabkommen auf, dann kämen Sockelvereinbarungen zu handels- und wirtschaftlicher Kooperation in Betracht. ${ }^{28}$ Im Sinne transaktionaler Politik könnte die Kooperation in jenen Bereichen ansetzen, in denen die Parteien jeweils Interessen haben oder gegeneinander verrechnen. Die sogenannte Erklärung EU-Türkei zur irregulären Migration wäre ein (aus der Not geborenes) Beispiel dafür. ${ }^{29}$ Themenbereiche wären also Fluchtbewegungen, reguläre Einwanderung und Personenmobilität, organisierte Kriminalität, Sicherheitssektor, Bildungspolitik, Entwicklungshilfe und Institutionenaufbau. Die EU müsste für sich klären, inwieweit sie im Rahmen von Sockelvereinbarungen besondere Konditionalitätsanforderungen aufstellt oder nicht.

\section{Konnektivitätsprovider}

Inspiriert von der offenbar attraktiven „Belt and Road“-Initiative Chinas könnte die EU sich auf die Finanzierung und Lancierung von Infrastrukturprojekten (Energie, Verkehr, Forschung) beschränken oder - anders gelagert - ihre Angebote im Bereich der technischen Hilfe und beim Institutionenaufbau erheblich ausbauen. Hier geht es um funktionale Kooperation mit der Erwartung, dass sich diese auch politisch auszahlt. Die EU hat im Kontext der Globalen Strategie für die Außen- und Sicherheitspolitik der Europäischen

26 Europäische Kommission: Kommission schlägt Modernisierung der Zollunion mit der Türkei vor, Pressemitteilung, 21. Dezember 2016, IP/16/4468; Ufuk Alkan: The Modernization of Turkey's Customs Union with the European Union: Reasons and Possible Outcomes, College of Europe: EU Diplomacy Paper 9/2017; Fadi Hakura: EU-Turkey Customs Union: Prospects for Modernization and Lessons for Brexit, Chatham House: Briefing Europe Programme Dezember 2018.

27 Unian Information Agency: Ukraine intends to join Customs Union with EU - Poroshenko, 13. Juli 2017.

28 Kai-Olaf Lang/Barbara Lippert: The EU and Its Neighbours. A Second Chance to Marry Democratisation and Stability, Stiftung Wissenschaft und Politik: SWP Comments 2/2012.

29 Europäischer Rat: Erklärung EU-Türkei, Pressemitteilung, 18. März 2016, 144/16. 
Union von 2016 und mit ihrem Ansatz für die Konnektivität zwischen Europa und Asien einen ausdrücklichen Zusammenhang zwischen Wohlstand, der EU und dem vernetzten Asien hergestellt. ${ }^{30}$ Dieser Ansatz soll keine Alternative zu dem der ÖP darstellen, vielmehr diesen Ländern eine zusätzliche Einbettung in regionale und sektorale Vernetzungsprojekte bieten. Es sei ein Ansatz, „der von den asiatischen Ländern, in unserer östlichen Nachbarschaft, auf dem westlichen Balkan und darüber hinaus gefragt ist“ “31

\section{Neue Grundformen: Teilmitgliedschaft und Europäischer Politik- und Wirtschaftsraum}

Eine neue Grundform für die Nachbarschaftsbeziehungen wäre die Teilmitgliedschaft. Bisher ließ die EU keine beliebige Kombination der Bauelemente von Partnerschaftsbeziehungen zu, sondern zog dafür rechtliche wie politische Grenzen. Diese konservative Position belegen die Eckpunkte der mit Großbritannien angestrebten Post-Brexit-Partnerschaft sehr deutlich (Tabelle 2). Sollte die EU die o.g. Integrationsgrenze jedoch aufheben und neben der (ggf. auch ausgeweiteten) Politikfeldintegration auch eine Teilhabemöglichkeit an EU-Institutionen und -Entscheidungen ermöglichen, rücken neue Grundformen in den Blick: Zunächst wäre die Idee der Junior-Mitgliedschaft zu nennen, mit der ein neuer Status für Mitglieder „Zweiter Klasse“ kreiert würde, die nicht über dieselben Rechte der Vertretung und Entscheidung in EU-Organen verfügen würden wie die „erstklassigen“ Länder. Das ginge über bisherige Formen der Differenzierung deutlich hinaus. Neben das bisher einzig gültige Konzept der Vollmitgliedschaft nach Art. 49 EUV träte das der Teilmitgliedschaft und innerhalb der EU kämen mehr oder weniger starr um einen Kern angeordnete Kreise unterschiedlicher Integrationsdichte hinzu. Es bedürfte dazu eines speziellen Aufnahmeverfahrens in Ergänzung zu Art. 49 EUV und eigener Kriterien für den Beitritt als Junior-Mitglied. ${ }^{32}$ Diese neue Option käme für Länder des Westbalkans infrage, die eine Fortsetzung oder gar Vervielfachung der Unterstützung erwarten und dafür ggf. eine Deklassierung in Kauf nähmen. Eine auf leistungsstarke Staaten zugeschnittene Variante könnte so ausfallen, wie sie für die kontinentale Partnerschaft ins Auge gefasst wurde. ${ }^{33}$ Bezeichnenderweise wird dieser Vorschlag, der die bislang geltenden Prinzipien der Beteiligung von Drittstaaten an Entscheidungsprozessen und deren Vertretung in EU-Organen über Bord wirft, von den Autoren nicht durchdekliniert. ${ }^{34}$ Das politische System der EU würde in der Folge dieser Teilmitgliedschaft weiter ausfransen, noch komplexer oder gar gesprengt werden.

Eine zweite neue Grundform würde auf länderübergreifende Assoziierungsräume abzielen. Mit Ausnahme des EWR hat die EU bilaterale Vertragswerke mit einzelnen Ländern geschaffen, aber nur gelegentlich und eher ergänzend multilaterale Arrangements initiiert, so im Falle des SAP, der ÖP und der Union für den Mittelmeerraum (UfM). Unmittelbar

30 Vgl. Europäische Kommission: Gemeinsame Mitteilung an das Europäische Parlament, den Rat, den Europäischen Wirtschafts- und Sozialausschuss, den Ausschuss der Regionen und die Europäische Investitionsbank. Förderung der Konnektivität zwischen Europa und Asien - Elemente einer EU-Strategie, JOIN(2018) 31 final.

31 Jyrki Katainen, zitiert in: Europäische Kommission, Vertretung in Deutschland: Europa und Asien verbinden: EU schlägt neue Strategie vor, 19. September 2018.

32 Duff: On Governing Europe, 2012.

33 Pisani-Ferry et al.: Europe after Brexit, 2016.

34 Ebenda. 
nach 1989 und bevor die EU sich auf die Erweiterung politisch festgelegt hatte, waren ordnungspolitische Ideen ventiliert worden, die auf länderübergreifende Konstruktionen neben der EU oder mit dieser mehr oder minder eng verbundene hinausliefen. Am bekanntesten waren die von François Mitterrand angeregte Gesamteuropäische Konföderation oder die rein funktional ausgerichtete Gesamteuropäische Aufgabenkonföderation. ${ }^{35}$ Mitterrands Vorschlag war als (vorübergehende oder dauerhafte) Alternative zur EU-Mitgliedschaft gedacht und damals postwendend von den ostmitteleuropäischen Regierungen abgelehnt worden. Ähnlich kritisch dürften heute Länder wie Georgien oder die Ukraine Vorschläge sehen, die auf länderübergreifende Organisationsformen abzielen.

Aus Sicht der EU bleibt es dennoch relevant, sich Gedanken über die Sicherheitsordnung des osteuropäischen Raums zu machen. Das ist auch deshalb von Bedeutung, weil die EU nicht von einer parallelen oder gar vorgängigen Expansion der NATO ausgehen kann, wie es beim Beitritt der ostmitteleuropäischen Staaten zu derselben und zur EU der Fall war. Ein Ausbau der Gemeinsamen Sicherheits- und Verteidigungspolitik (GSVP) könnte einerseits attraktiv für die ÖP-Länder sein, für die ein NATO-Beitritt nicht in Sicht ist. Aber zugleich dürfte das gerade aus russischer Sicht die Integrationskonkurrenz zwischen Moskau und Brüssel befeuern. Die EU-Mitgliedstaaten sind sowohl im Hinblick auf die NATO-Frage als auch den Ausbau der ÖP in diese Richtung uneins, wobei die Linie hier tendenziell zwischen den Ländern im Norden und Osten einerseits und denen im Westen und Süden andererseits verläuft. ${ }^{36}$

Zunächst könnte die EU das nicht ausgeschöpfte Potenzial des Art. 8 EUV ins Auge fassen. Dieser sieht vor, dass die EU „besondere Beziehungen zu den Ländern in ihrer Nachbarschaft [entwickelt], um einen Raum des Wohlstands und der guten Nachbarschaft zu schaffen, der auf den Werten der Union aufbaut und sich durch enge, friedliche Beziehungen auf der Grundlage der Zusammenarbeit auszeichnet“. Platziert im Titel „Gemeinsame Bestimmungen“ eröffnet Art. 8 einen politisch-normativen Horizont für die EU als Ordnungsmacht; dagegen ist die Assoziierung neutral formuliert. Was Art. 8 unter „Raum“ versteht, ist äußerst vage und ebenso offenbleibt, wie dieser zu gestalten ist. Das lässt andererseits einigen politischen Spielraum: So könnten multilaterale Formate (ÖP, UfM) und Vorschläge, die z.B. in Richtung Konföderation als Ausdruck politischer Zusammengehörigkeit gehen oder die die Zusammenarbeit mit anderen Organisationen (NATO, Europarat, Organisation für Sicherheit und Zusammenarbeit in Europa, Eurasische Wirtschaftsunion) herausstellen, an Art. 8 gebunden und damit auch seitens der EU politisch profilierter werden. Kritisch wäre hier einzuwenden, dass die EU mit einem solchen Ansatz (ähnlich wie bei der ENP) wenig Mehrwert für die Zielländer und auch kein politisches Momentum für die eigene strategischere Ausrichtung gegenüber diesem Raum schafft.

35 François Mitterrand: Ansprache des französischen Staatspräsidenten auf der Schlußsitzung der Tagung zur Europäischen Konföderation in Prag am 14. Juni 1991 (Auszüge), in: Europa-Archiv 15-16/1991, S. D 390ff.; Europäisches Parlament: Bericht für den Institutionellen Ausschuss des Europäischen Parlaments. „Über die Gestaltung und Strategie der Europäischen Union im Hinblick auf ihre Erweiterung und Schaffung einer gesamteuropäischen Ordnung“. Sitzungsdokument A3.0189/92 (Bericht Hänsch), 21. Mai 1992, Punkte 8-12; Lippert: Teilhabe statt Mitgliedschaft?, 2007, S. 92ff.; Jean Musitelli: François Mitterrand, architecte de la Grande Europe: le project de Confédération européenne (1990-1991), in: Revue Internationale et Stratégique 2/2011, S. $18 \mathrm{ff}$.

36 Barbara Lippert/Nicolai von Ondarza/Volker Perthes (Hrsg.): Strategische Autonomie Europas. Akteure, Handlungsfelder, Zielkonflikte, Stiftung Wissenschaft und Politik: SWP-Studie 2/2019, S. $33 f$. 
Weitergehend und konkreter wäre der Vorschlag eines Europäischen Politik- und Wirtschaftsraums (EPWR). Dessen explizites Ziel wäre es, ungefestigten osteuropäischen Transformationsstaaten, die sich in einem prekären Sicherheitsumfeld befinden und mittelfristig weder auf eine EU- noch eine NATO-Mitgliedschaft bauen können, eine sichtbare Anbindung an das demokratische Europa zu ermöglichen. Der EPWR würde im Sinne des Art. 8 einen Raum des Wohlstands und der guten Nachbarschaft anstreben. Er wäre nicht einfach ein „EWR 2.0“. Denn der EWR stellt die funktionale und auf den Binnenmarkt begrenzte Integration in den Vordergrund. Er schafft einen einheitlichen Regulierungsraum, spielt jedoch die politische und gemeinschaftliche Dimension eher herunter. Beim EPWR müsste hingegen das politische Profil besonders stark und sichtbar sein, um überhaupt einen Mehrwert gegenüber den bilateralen Beziehungen zu schaffen. Für einen EPWR wäre die heutige multilaterale ÖP ein möglicher Ausgangspunkt. Denn sie könnte über eine Clearingstelle und Kooperationsplattform für multilaterale und regionale Vorhaben weiterentwickelt und politisch profiliert werden. ${ }^{37}$ Wollte die EU diesen Weg einschlagen, müsste sie sich mit einer Vielzahl von grundsätzlichen und spezielleren Fragen befassen: Soll es im EPWR (neben dem EU-Pfeiler) einen DCFTA/ÖP-Pfeiler geben (analog zum EFTA-Pfeiler im EWR)? Soll der EPWR also eine Region-building-Komponente enthalten? Ist eine Ausgangsaufstellung mit nur einem DCFTA-Land möglich? Gibt es neben der Freiwilligkeit besondere (politische und wirtschaftliche) Qualifizierungskriterien? Ist der EPWR ein überwölbendes Format oder ersetzt er die bilateralen DCFTA im Sinne eines Upgradings? Was ist der inhaltliche Umfang? Welche gemeinsamen Entscheidungsverfahren werden vorgesehen? Antworten auf diese Fragen sind sinnvollerweise vor dem Hintergrund zu treffen, inwieweit sich die EU in Richtung strategischer Autonomie bewegt und wie sie ihre Politik gegenüber Russland ausrichtet. Russland war der Dreh- und Angelpunkt der traditionellen Überlegungen zu einer gesamteuropäischen Freihandelszone oder einem Wirtschaftsraum von Lissabon bis Wladiwostok. Die Idee eines EPWR löst sich von der Moskau-zentrierten Sichtweise.

Angesichts der antagonistischen Grundkonstellation zwischen Russland und der EU, aber auch angesichts des Differenzierungsgrads unter den Ländern der ÖP ist es fraglich, ob ein inklusives, überwölbendes Format wie der skizzierte EPWR überhaupt noch in die politische Landschaft passt. Denn die implizite Ordnungsidee von homogenen Räumen oder gar von Gemeinschaftsbildung scheint obsolet. Man beachte nur die zentrifugalen Tendenzen innerhalb der EU. Die Ordnungsidee der Zukunft könnte eher die der Netzwerke und funktionalen, lose verkoppelten Teilordnungen sein. Dafür könnte sich die EU als eine Art Sammel- und Clearingstelle, eher als ein Dienstleister für Konnektivität denn als eine Ordnungsmacht anbieten.

\section{Variable Assoziierungsmodelle im Hinblick auf gesamteuropäische Ordnungsprobleme}

Obwohl die EU selbst immer weniger als Strategiegemeinschaft nach außen auftritt, bildet sie einstweilen noch ein politisch-wirtschaftliches Kraftfeld, auf das sich die Nachbarn

37 Allerdings hat die ÖP einen inklusiven Zuschnitt, der alle sechs osteuropäischen Länder unter einem Dach vereint (mit leichten Spezifika für Belarus). Zudem hat die EU ihre ursprüngliche Idee nicht widerrufen, dass prinzipiell auch eine Beteiligung Russlands an spezifischen Projekten möglich wäre. 
freiwillig und/oder mangels Alternative ausrichten. So hat die von Russland initiierte Eurasische Wirtschaftsunion keine Anziehungskraft im postsowjetischen Raum entwickelt. Ob im umfassenden Sinne oder primär wirtschaftlich - die EU steht im Zentrum schwelender gesamteuropäischer Ordnungsprobleme.

Nur wenige Assoziierte werden in den nächsten Jahren von der Teilintegration in die Mitgliedschaft wechseln können oder wollen. Entweder hapert es an ihrer Beitrittsreife oder das Beitrittsinteresse fehlt. Die EU wollte sich ihrerseits eine Atempause verschaffen, als sie die Beitrittsperspektive der Westbalkanländer politisch akzeptierte (jedoch nicht auf die osteuropäischen Länder erweiterte), aber zugleich die eigene Aufnahmefähigkeit zu einer wichtigen Voraussetzung für künftige Erweiterungen machte. Die Lage in der EU und in der Nachbarschaft hat sich seit diesem sogenannten Erweiterungskonsens von 2006 eher verschlechtert denn verbessert. ${ }^{38}$ Zum einen schwinden die Bindungskräfte im Innern der EU, die sie durch Formen differenzierter Integration zu bändigen oder produktiv zu gestalten versucht. Die EU hat bereits heute Mitglieder, die die Kopenhagener Beitrittskriterien nicht mehr erfüllen und gegen die sie keine durchgreifende Handhabe hat. Mit einer stabilitätspolitisch motivierten Aufnahme von „unreifen“ Westbalkanländern riskiert die EU, weiter an Glaubwürdigkeit und Funktionsfähigkeit zu verlieren. Zum anderen hat sich auch das Umfeld verschlechtert: Denn mit Großbritannien und der Türkei entfernen sich zwei strategisch wichtige Länder von der EU, während osteuropäische Staaten in Richtung EU drängen, von der sie Wohlstand und Schutz erwarten.

Vor dem Hintergrund der hier skizzierten etablierten Assoziierungsmodelle und neuer Grundformen ergibt sich folgendes Bild: Der EWR ist bis dato die einzige erfolgreiche Neugründung, die Parität zwischen der EU und einer Verbindung von Drittstaaten herstellt und diesen die Integration in den Binnenmarkt bietet. Anders als der EU-Pfeiler, der mit jeder Erweiterungsrunde größer wurde, ist der EFTA-Pfeiler unverändert geblieben. Die EFTA-Länder im EWR haben eine Erweiterung um Großbritannien zur Lösung des Brexit-Problems nicht aktiv gesucht. Es ist deshalb sehr wahrscheinlich, dass die EU für konsolidierte Demokratien mit funktionierender Marktwirtschaft spezielle bilaterale Arrangements aus den Standardangeboten der Assoziierung heraus entwickeln und zugleich die Tür für den Beitritt offen halten wird. Auch gegenüber der Türkei sowie den Ländern des Westbalkans und Osteuropas dürfte die EU ihre Politik der bilateralen Assoziierungsbeziehungen fortsetzen, die sie mit unterschiedlich starken Transformationskomponenten und Unterstützungspaketen anreichert. Die Qualität dieser bilateralen und differenziert ausgestalteten Beziehungen hängt nicht zuletzt von der jeweiligen politischen Responsivität der Regierungen und Gesellschaften sowie von der Implementierungsstärke der Zielländer ab. Diese Assoziierungsbeziehungen könnten zu einer maximalen Teilintegration, etwa bei der Strukturpolitik führen, bevor oder ohne dass die Länder EU-Mitglied würden. Denn die etablierten Assoziierungsmodelle der EU sind anpassungs- und ausbaufähig. Wäre die geopolitische Großwetterlage freundlich, könnte die EU auf diesen eingeschlagenen Pfaden beharrlich und kraftvoll weitergehen. Da das aber nicht der Fall ist, müsste sie sich strategischer verhalten, indem sie Antworten auf die hier diskutierten Optionen und Herausforderungen findet.

38 Rat der Europäischen Union: Europäischer Rat (Brüssel). 14./15. Dezember 2006. Schlussfolgerungen des Vorsitzes. 